\title{
Data Services and Trans-National Access Program Accessible Through EPOS Multi-Scale Laboratories
}

E. Hellebrand*, R.J.F. Wessels, M.F. HAMERS, O.A. LANGe, G. TER MAAT, O. PluemPer, \& M. DRURY

Utrecht University, Department of Earth Science, Utrecht, The Netherlands (e.w.g.hellebrand@uu.nl)

The Multi-scale Laboratories (MSL) form one of the Thematic Core Services (TCS) of the European Plate Observing System (EPOS), an infrastructure project supported by the European Union. Participants and collaborating laboratories from Belgium, Bulgaria, France, Germany, Italy, the Netherlands, Norway, Portugal, Spain, Switzerland, and the UK are already organized in the TCS MSL. Unaffiliated European solid Earth sciences laboratories are welcome and encouraged to join the growing TCS MSL community.

Utrecht University plays a key role in two aspects of the TCS MSL: (1) designing and implementing the IT framework for curating and sharing microstructural and associated data and metadata acquired in the field, experimental laboratories, and by a broad spectrum of analytical in-situ methods. The TCS MSL is committed to making Earth science laboratory data Findable, Accessible, Interoperable, and Reusable (FAIR). To support this commitment we have developed an online portal that brings together DOI-referenced data publications from research data repositories related to the TCS MSL context (https://epos-msl.uu.nl/).

(2) In addition, TCS MSL offers a Trans-National Access (TNA) program that allows researchers to apply for access to the participating EPOS MSL laboratories. Three pilot calls were launched in 2017, 2018, and 2019, with a fourth round scheduled for 2020. The pilot calls were used to develop and refine the EPOS wide TNA principles and to initialize an EPOS brokering service, where information on each facility offering access will be available for the user and where calls for proposals are advertised. Access to the participating laboratories is currently supported by national funding. Based on the EPOS Data policy \& TNA General Principles, access to the laboratories is regulated by common rules and a transparent policy, including procedures and mechanisms for application, negotiation, proposal evaluation, user feedback, use of laboratory facilities and data curation. The upgraded analytical facilities at Utrecht University include a full range of state-of-the-art SEM (Zeiss EVO and Gemini, FEI Helios FIB-SEM) and TEM (FEI T20's, Arctica, and Spectre) instruments, as well as a high-resolution X-ray microscope (Zeiss Xradia 610 Versa). 